\title{
QUANTITATIVE WEIGHTED ESTIMATES FOR THE LITTLEWOOD-PALEY SQUARE FUNCTION AND MARCINKIEWICZ MULTIPLIERS
}

\author{
ANDREI K. LERNER
}

\begin{abstract}
Quantitative weighted estimates are obtained for the Littlewood-Paley square function $S$ associated with a lacunary decomposition of $\mathbb{R}$ and for the Marcinkiewicz multiplier operator. In particular, we find the sharp dependence on $[w]_{A_{p}}$ for the $L^{p}(w)$ operator norm of $S$ for $1<p \leqslant 2$.
\end{abstract}

\section{Introduction}

Given a weight $w$ (i.e., a non-negative locally integrable function on $\mathbb{R}^{n}$ ), we say that $w \in A_{p}, 1<p<\infty$, if

$$
[w]_{A_{p}}=\sup _{Q}\langle w\rangle_{Q}\left\langle w^{1-p^{\prime}}\right\rangle_{Q}^{p-1}<\infty,
$$

where the supremum is taken over all cubes $Q \subset \mathbb{R}^{n}$ and $\langle\cdot\rangle_{Q}$ is the integral mean over $Q$.

In the recent decade, it has been of great interest to obtain the $L^{p}(w)$ operator norm estimates (possibly optimal) in terms of $[w]_{A_{p}}$ for the different operators in harmonic analysis. In particular, it was established that the $L^{p}(w)$ operator norms for Calderón-Zygmund and a large class of Littlewood-Paley operators are bounded by a multiple of $[w]_{A_{p}}^{\max \left(1, \frac{1}{p-1}\right)}$ and $[w]_{A_{p}}^{\max \left(\frac{1}{2}, \frac{1}{p-1}\right)}$, respectively, and these bounds are sharp for all $1<p<\infty$ (see [20, 11, 6, 16]).

On the other hand, there are still a number of operators for which the sharp bounds in terms of $[w]_{A_{p}}$ are not known yet. For example, for rough homogeneous singular integrals $T_{\Omega}$ with angular part $\Omega \in L^{\infty}$ the currently best known result says that $\left\|T_{\Omega}\right\|_{L^{2}(w) \rightarrow L^{2}(w)}$ is at most a multiple of $[w]_{A_{2}}^{2}$, and it is an open question whether this bound is sharp (see [5, 14, 17]). Several other examples are the main objects of the present paper.

2010 Mathematics Subject Classification. 42B20, 42B25.

Key words and phrases. Square function, Marcinkiewicz multipliers, weighted norm inequalities.

The author was supported by ISF grant No. 447/16 and ERC Starting Grant No. 713927. 
We consider the classical Littlewood-Paley square function associated with a lacunary decomposition of $\mathbb{R}$ and the Marcinkiewicz multiplier operator. Recall the definitions of these objects. For $k \in \mathbb{Z}$ set $\Delta_{k}=\left(-2^{k+1},-2^{k}\right] \cup\left[2^{k}, 2^{k+1}\right)$. The Littlewood-Paley square function we shall deal with is defined by

$$
S f=\left(\sum_{k \in \mathbb{Z}}\left|S_{\Delta_{k}} f\right|^{2}\right)^{1 / 2}
$$

where $\widehat{S_{\Delta_{k}} f}=\widehat{f} \chi_{\Delta_{k}}$. We say that $T_{m}$ is the Marcinkiewicz multiplier operator if $\widehat{T_{m} f}=m \widehat{f}$, where $m \in L^{\infty}$ and

$$
\sup _{k \in \mathbb{Z}} \int_{\Delta_{k}}\left|m^{\prime}(t)\right| d t<\infty .
$$

The fact that $S$ and $T_{m}$ are bounded on $L^{p}(w)$ for $w \in A_{p}$ is well known and due to D. Kurtz [15]. Tracking the dependence on $[w]_{A_{p}}$ in the known proofs yields, for example, that the $L^{2}(w)$ operator norms of $S$ and $T_{m}$ are bounded by a multiple of $[w]_{A_{2}}^{2}$ and $[w]_{A_{2}}^{4}$, respectively.

In this paper we give new proofs of the $L^{p}(w)$ boundedness of $S$ and $T_{m}$ providing better quantitative estimates it terms of $[w]_{A_{p}}$. Our main results are the following.

Theorem 1.1. If $\alpha_{p}$ is the best possible exponent in

$$
\|S\|_{L^{p}(w) \rightarrow L^{p}(w)} \leqslant C_{p}[w]_{A_{p}}^{\alpha_{p}}
$$

then

$$
\max \left(1, \frac{3}{2} \frac{1}{p-1}\right) \leqslant \alpha_{p} \leqslant \frac{1}{2} \frac{1}{p-1}+\max \left(1, \frac{1}{p-1}\right) \quad(1<p<\infty) ;
$$

in particular, $\alpha_{p}=\frac{3}{2} \frac{1}{p-1}$ for $1<p \leqslant 2$.

Theorem 1.2. If $\beta_{p}$ is the best possible exponent in

$$
\left\|T_{m}\right\|_{L^{p}(w) \rightarrow L^{p}(w)} \leqslant C_{p, m}[w]_{A_{p}}^{\beta_{p}},
$$

then

$$
\frac{3}{2} \max \left(1, \frac{1}{p-1}\right) \leqslant \beta_{p} \leqslant \frac{p^{\prime}}{2}+\max \left(1, \frac{1}{p-1}\right) \quad(1<p<\infty) .
$$

Observe that the lower bounds for $\alpha_{p}$ and $\beta_{p}$ are immediate consequences of several known results. By a general extrapolation argument due to T. Luque, C. Pérez and E. Rela [19], if an operator $T$ is such that its unweighted $L^{p}$ norms satisfy $\|T\|_{L^{p} \rightarrow L^{p}} \simeq \frac{1}{(p-1)^{\gamma_{1}}}$ as $p \rightarrow 1$ and $\|T\|_{L^{p} \rightarrow L^{p}} \simeq p^{\gamma_{2}}$ as $p \rightarrow \infty$, then the best possible exponent $\xi_{p}$ in $\|T\|_{L^{p}(w) \rightarrow L^{p}(w)} \leqslant C[w]_{A_{p}}^{\xi_{p}}$ satisfies $\xi_{p} \geqslant \max \left(\gamma_{2}, \frac{\gamma_{1}}{p-1}\right)$. Therefore, the 
lower bounds for $\alpha_{p}$ and $\beta_{p}$ follow from the sharp unweighted behavior of the $L^{p}$ norms of $S$ and $T_{m}$.

Such a behavior for $S$ was found by J. Bourgain [3]:

$$
\|S\|_{L^{p} \rightarrow L^{p}} \simeq \frac{1}{(p-1)^{3 / 2}} \text { as } p \rightarrow 1 \text { and }\|S\|_{L^{p} \rightarrow L^{p}} \simeq p \text { as } p \rightarrow \infty,
$$

which implies the lower bound for $\alpha_{p}$. These asymptotic relations were obtained in 3 for the circle version of the Littlewood-Paley square function but the arguments can be transferred to the real line version in a straightforward way. An alternative proof of the first asymptotic relation in (1.1) has been recently found by O. Bakas [1.

The sharp unweighted $L^{p}$ norm behavior of $T_{m}$ is due to T. Tao and J. Wright [21]:

$$
\left\|T_{m}\right\|_{L^{p} \rightarrow L^{p}} \simeq \max \left(p, p^{\prime}\right)^{3 / 2} \quad(1<p<\infty),
$$

which implies the lower bound for $\beta_{p}$.

Bourgain's proof [3] of the first relation in (1.1) was based on a dual restatement in terms of the vector-valued operator $\sum_{k \in \mathbb{Z}} S_{\Delta_{k}} \psi_{k}$ with its subsequent handling by means of the Chang-Wilson-Wolff inequality [4]. Our proof of the upper bound for $\alpha_{p}$ follows similar ideas but with some modifications. As the key tool we use Theorem 2.7, which is a discrete analogue of the sharp weighted continuous square function estimate proved by M. Wilson [22]. Notice that the latter estimate is also based on the Chang-Wilson-Wolff inequality. We mention that the sharp $L^{2}(w)$ bound in Theorem 1.1,

$$
\|S\|_{L^{2}(w) \rightarrow L^{2}(w)} \leqslant C[w]_{A_{2}}^{3 / 2},
$$

by extrapolation yields yet another proof of the unweighted upper bound $\|S\|_{L^{p} \rightarrow L^{p}} \leqslant \frac{C}{(p-1)^{3 / 2}}, 1<p \leqslant 2$ (see Remark 4.2 below).

Another important ingredient used both in the proofs of Theorems 1.1 and 1.2 is Lemma 3.2. This lemma establishes a two-weighted estimate for the multiplier operator $T_{m \chi_{[a, b]}}$. The need to consider two-weighted estimates comes naturally from the method of the proof of Theorem 1.2 .

The paper is organized as follows. Section 2 contains some preliminaries and, in particular, the proof of Theorem 2.7. In Section 3 we prove two main technical lemmas. The proof of Theorems 1.1 and 1.2 is contained in Section 4. In Section 5 we make several conjectures related to the sharp upper bounds for $\alpha_{p}$ and $\beta_{p}$.

\section{Preliminaries}

Although the main objects we deal with are defined on $\mathbb{R}$, the results of subsections $2.1,2.2$ and 2.3 are valid on $\mathbb{R}^{n}$. 
2.1. Dyadic lattices. The material of this subsection is taken from [18]. Given a cube $Q_{0} \subset \mathbb{R}^{n}$, let $\mathcal{D}\left(Q_{0}\right)$ denote the set of all dyadic cubes with respect to $Q_{0}$, that is, the cubes obtained by repeated subdivision of $Q_{0}$ and each of its descendants into $2^{n}$ congruent subcubes.

Definition 2.1. A dyadic lattice $\mathscr{D}$ in $\mathbb{R}^{n}$ is any collection of cubes such that

(i) if $Q \in \mathscr{D}$, then each child of $Q$ is in $\mathscr{D}$ as well;

(ii) every 2 cubes $Q^{\prime}, Q^{\prime \prime} \in \mathscr{D}$ have a common ancestor, i.e., there exists $Q \in \mathscr{D}$ such that $Q^{\prime}, Q^{\prime \prime} \in \mathcal{D}(Q)$;

(iii) for every compact set $K \subset \mathbb{R}^{n}$, there exists a cube $Q \in \mathscr{D}$ containing $K$.

In order to construct a dyadic lattice $\mathscr{D}$, it suffices to fix an arbitrary cube $Q_{0}$ and to expand it dyadically (carefully enough in order to cover the whole space) by choosing one of $2^{n}$ possible parents for the top cube and including it into $\mathscr{D}$ together with all its dyadic subcubes during each step. Therefore, given $h>0$, one can choose a dyadic lattice $\mathscr{D}$ such that for any $Q \in \mathscr{D}$ its sidelength $\ell_{Q}$ will be of the form $2^{k} h, k \in \mathbb{Z}$.

Theorem 2.2. (The Three Lattice Theorem) For every dyadic lattice $\mathscr{D}$, there exist $3^{n}$ dyadic lattices $\mathscr{D}^{(1)}, \ldots, \mathscr{D}^{\left(3^{n}\right)}$ such that

$$
\{3 Q: Q \in \mathscr{D}\}=\bigcup_{j=1}^{3^{n}} \mathscr{D}^{(j)}
$$

and for every cube $Q \in \mathscr{D}$ and $j=1, \ldots, 3^{n}$, there exists a unique cube $R \in \mathscr{D}^{(j)}$ of sidelength $\ell_{R}=3 \ell_{Q}$ containing $Q$.

2.2. Some Littlewood-Paley theory. Denote by $\mathscr{S}\left(\mathbb{R}^{n}\right)$ the class of Schwartz functions on $\mathbb{R}^{n}$. The following statement can be found in [10, Lemma 5.12] (see also [9, p. 783] for some details).

Lemma 2.3. There exist $\varphi, \theta \in \mathscr{S}\left(\mathbb{R}^{n}\right)$ satisfying the following properties:

(i) $\operatorname{supp} \theta \subset\{x:|x| \leqslant 1\}$ and $\int \theta=0$;

(ii) $\operatorname{supp} \hat{\varphi} \subset\{\xi: 1 / 2 \leqslant|\xi| \leqslant 2\}$;

(iii) $\sum_{k \in \mathbb{Z}} \widehat{\varphi}\left(2^{-k} \xi\right) \hat{\theta}\left(2^{-k} \xi\right) \equiv 1$ for all $\xi \neq 0$.

Property (iii) implies, by taking the Fourier transform, the discrete version of the Calderón reproducing formula:

$$
f=\sum_{k \in \mathbb{Z}} f * \varphi_{2^{-k}} * \theta_{2^{-k}} .
$$


Remark 2.4. There are several interpretations of convergence in (2.1). In particular, we will use the following one. Let $1<p<\infty$ and suppose $w \in A_{p}$. Given $f \in L^{p}(w)$ and $N \in \mathbb{N}$, set

$$
f_{N}(x)=\sum_{k=-N}^{N} \int_{E_{N}}\left(f * \varphi_{2^{-k}}\right)(y) \theta_{2^{-k}}(x-y) d y,
$$

where $\left\{E_{N}\right\}$ is an increasing sequence of bounded measurable sets such that $E_{N} \rightarrow \mathbb{R}^{n}$. Then $f_{N} \rightarrow f$ in $L^{p}(w)$ as $N \rightarrow \infty$. For the continuous version of (2.1) this fact was proved by M. Wilson [23, Th. 7.1] (see also [24]), and in the discrete case the proof follows the same lines.

The following result is also due to M. Wilson (see [22, Lemma 2.3] and [23, Th. 4.3]).

Theorem 2.5. Let $\mathscr{D}$ be a dyadic lattice and let $\mathscr{G} \subset \mathscr{D}$ be a finite family of cubes. Assume that $f=\sum_{Q \in \mathscr{G}} \lambda_{Q} a_{Q}$, where $\operatorname{supp} a_{Q} \subset Q$, $\left\|a_{Q}\right\|_{L^{\infty}} \leqslant|Q|^{-1 / 2},\left\|\nabla a_{Q}\right\|_{L^{\infty}} \leqslant \ell_{Q}^{-1}|Q|^{-1 / 2}$ and $\int a_{Q}=0$. Then for all $1<p<\infty$ and for every $w \in A_{p}$,

$$
\|f\|_{L^{p}(w)} \leqslant C_{p, n}[w]_{A_{p}}^{1 / 2}\left\|\left(\sum_{Q \in \mathscr{G}} \frac{\left|\lambda_{Q}\right|^{2}}{|Q|} \chi_{Q}\right)^{1 / 2}\right\|_{L^{p}(w)} .
$$

Remark 2.6. Notice that actually (2.2) was proved in [22] with a smaller $[w]_{A_{\infty}}$ constant defined by

$$
[w]_{A_{\infty}}=\sup _{Q} \frac{1}{\int_{Q} w} \int_{Q} M\left(w \chi_{Q}\right)
$$

where $M f(x)=\sup _{Q \ni x} \frac{1}{|Q|} \int_{Q}|f|$ is the Hardy-Littlewood maximal operator. See also [13] for various estimates in terms of $[w]_{A_{\infty}}$.

Theorem 2.5] along with the continuous version of (2.1) was applied in 22 in order to obtain the $L^{p}(w)$-norm relation between $f$ and the continuous square function. In a similar way, using (2.1), we obtain the $L^{p}(w)$-norm relation between $f$ and the discrete square function defined (for a given dyadic lattice $\mathscr{D}$ ) by

$$
S_{\varphi, \mathscr{D}}(f)(x)=\left(\sum_{k \in \mathbb{Z}} \sum_{Q \in \mathscr{D}: \ell_{Q}=2^{-k}}\left(\frac{1}{|Q|} \int_{Q}\left|f * \varphi_{2^{-k}}\right|^{2}\right) \chi_{Q}(x)\right)^{1 / 2} .
$$

Theorem 2.7. There exists a function $\varphi \in \mathscr{S}\left(\mathbb{R}^{n}\right)$ with $\operatorname{supp} \hat{\varphi} \subset\{\xi$ : $1 / 2 \leqslant|\xi| \leqslant 2\}$ and there are $3^{n}$ dyadic lattices $\mathscr{D}^{(j)}$ such that for every 
$w \in A_{p}$ and for any $f \in L^{p}(w), 1<p<\infty$,

$$
\|f\|_{L^{p}(w)} \leqslant C_{p, n}[w]_{A_{p}}^{1 / 2} \sum_{j=1}^{3^{n}}\left\|S_{\varphi, \mathscr{D}(j)}(f)\right\|_{L^{p}(w)} .
$$

Proof. Let $\varphi, \theta$ be functions from Lemma 2.3. Let $\mathscr{D}$ be a dyadic lattice such that for every $Q \in \mathscr{D}$ its sidelength is of the form $\ell_{Q}=\frac{2^{k}}{3}, k \in \mathbb{Z}$. Let $\mathscr{D}^{(j)}, j=1, \ldots, 3^{n}$, be dyadic lattices obtained by applying Theorem 2.2 to $\mathscr{D}$. Then for every $Q \in \mathscr{D}^{(j)}$ its sidelength is of the form $\ell_{Q}=2^{k}, k \in \mathbb{Z}$.

For $Q \in \mathscr{D}$ with $\ell_{Q}=2^{-k} / 3$ set

$$
\gamma_{Q}(x)=\int_{Q}\left(f * \varphi_{2^{-k}}\right)(y) \theta_{2^{-k}}(x-y) d y .
$$

It is easy to check that $\operatorname{supp} \gamma_{Q} \subset 3 Q, \int \gamma_{Q}=0$ and

$$
\max \left(\left\|\gamma_{Q}\right\|_{L^{\infty}}, \ell_{Q}\left\|\nabla \gamma_{Q}\right\|_{L^{\infty}}\right) \leqslant c\left(\frac{1}{|Q|} \int_{Q}\left|f * \varphi_{2^{-k}}\right|^{2}\right)^{1 / 2},
$$

where $c$ depends only on $n$ and $\theta$.

Take an increasing sequence of cubes $Q_{N} \in \mathscr{D}$ such that $\ell_{Q_{N}}=$ $\frac{2^{N}}{3}, N \in \mathbb{N}$. Set

$$
\mathscr{G}_{N}=\left\{Q \in \mathscr{D}: Q \subseteq Q_{N}, \ell_{Q}=2^{-k} / 3, k \in[-N, N]\right\} .
$$

By Theorem 2.2, one can write

$$
\left\{3 Q: Q \in \mathscr{G}_{N}\right\}=\bigcup_{j=1}^{3^{n}} \mathscr{G}_{N}^{(j)},
$$

where $\mathscr{G}_{N}^{(j)} \subset \mathscr{D}^{(j)}$. Then

$$
\begin{aligned}
f_{N}(x) & =\sum_{k=-N}^{N} \int_{Q_{N}}\left(f * \varphi_{2^{-k}}\right)(y) \theta_{2^{-k}}(x-y) d y \\
& =\sum_{k=-N}^{N} \sum_{Q \in \mathscr{D}: Q \subseteq Q_{N}, \ell_{Q}=2^{-k} / 3} \gamma_{Q}(x)=\sum_{j=1}^{3^{n}} \sum_{P \in \mathscr{G}_{N}^{(j)}} \lambda_{P}^{(j)} a_{P}^{(j)}
\end{aligned}
$$

where, for $P=3 Q, Q \in \mathscr{D}, \ell_{Q}=2^{-k} / 3$, we set

$$
\lambda_{P}^{(j)}=c\left(\int_{3 Q}\left|f * \varphi_{2^{-k}}\right|^{2}\right)^{1 / 2}
$$

and $a_{P}^{(j)}=\frac{1}{\lambda_{P}^{(j)}} \gamma_{Q}$ 
By (2.3), we have that the functions $a_{P}^{(j)}$ satisfy all conditions from Theorem 2.5. Therefore, by (2.2),

$$
\begin{aligned}
\left\|f_{N}\right\|_{L^{p}(w)} & \leqslant C_{p, n}[w]_{A_{p}}^{1 / 2} \sum_{j=1}^{3^{n}}\left\|\left(\sum_{P \in \mathscr{G}_{N}^{(j)}} \frac{\left|\lambda_{P}^{(j)}\right|^{2}}{|P|} \chi_{P}\right)^{1 / 2}\right\|_{L^{p}(w)} \\
& \leqslant C_{p, n}[w]_{A_{p}}^{1 / 2} \sum_{j=1}^{3^{n}}\left\|S_{\varphi, \mathscr{D}^{(j)}}(f)\right\|_{L^{p}(w)} .
\end{aligned}
$$

Applying the convergence argument as described in Remark 2.4 completes the proof.

2.3. The sharp extrapolation. The following result was proved in [8].

Theorem 2.8. Assume that for some $f, g$ and for all weights $w \in A_{p_{0}}$,

$$
\|f\|_{L^{p_{0}}(w)} \leqslant C N\left([w]_{A_{p_{0}}}\right)\|g\|_{L^{p_{0}}(w)}
$$

where $N$ is an increasing function and the constant $C$ does not depend on $w$. Then for all $1<p<\infty$ and all $w \in A_{p}$,

$$
\|f\|_{L^{p}(w)} \leqslant C K(w)\|g\|_{L^{p}(w)},
$$

where

$$
K(w)= \begin{cases}N\left([w]_{A_{p}}\left(2\|M\|_{L^{p}(w) \rightarrow L^{p}(w)}\right)^{p_{0}-p}\right), & \text { if } p<p_{0} \\ N\left([w]_{A_{p}}^{\frac{p_{0}-1}{p-1}}\left(2\|M\|_{L^{p^{\prime}}\left(w^{1-p^{\prime}}\right) \rightarrow L^{p^{\prime}}\left(w^{1-p^{\prime}}\right)}\right)^{\left.\frac{p-p_{0}}{p-1}\right),}\right. & \text { if } p>p_{0} .\end{cases}
$$

In particular, $K(w) \leqslant C_{1} N\left(C_{2}[w]_{A_{p}}^{\max }\left(1, \frac{p_{0}-1}{p-1}\right)\right)$ for $w \in A_{p}$.

\subsection{Some two-weighted estimates. Let}

$$
H f(x)=\operatorname{p.v} \cdot \frac{1}{\pi} \int_{\mathbb{R}} \frac{f(y)}{x-y} d y \text { and } H^{\star} f(x)=\sup _{\varepsilon>0} \frac{1}{\pi}\left|\int_{|x-y|>\varepsilon} \frac{f(y)}{x-y} d y\right|
$$

be the Hilbert and the maximal Hilbert transforms, respectively.

Given two weights $u$ and $v$, set

$$
[u, v]_{A_{2}}=\sup _{Q}\langle u\rangle_{Q}\left\langle v^{-1}\right\rangle_{Q}
$$

Then the following two-weighted estimates hold:

$$
\begin{aligned}
& \max \left(\|M\|_{L^{2}(v) \rightarrow L^{2}(u)},\left\|H^{\star}\right\|_{L^{2}(v) \rightarrow L^{2}(u)}\right) \\
& \leqslant C[u, v]_{A_{2}}^{1 / 2}\left([u]_{A_{2}}^{1 / 2}+[v]_{A_{2}}^{1 / 2}\right) .
\end{aligned}
$$

The proofs of these estimates can be found in [12, 13] (notice that stronger versions of (2.4) in terms of the $[w]_{A_{\infty}}$ constants are proved there). 
2.5. The partial sum operator. Given an interval $[a, b]$, the partial sum operator $S_{[a, b]}$ is defined by $\widehat{S_{[a, b]} f}=\widehat{f} \chi_{[a, b]}$. We will use two standard facts about $S_{[a, b]}$ (see, e.g., [7]). First,

$$
S_{[a, b]}=\frac{i}{2}\left(\mathcal{M}_{a} H \mathcal{M}_{-a}-\mathcal{M}_{b} H \mathcal{M}_{-b}\right),
$$

where $\mathcal{M}_{a} f(x)=e^{2 \pi i a x} f(x)$. Second, if $\left(T_{m \chi_{[a, b]}} f\right)^{\wedge}=m \chi_{[a, b]} \widehat{f}$, then

$$
T_{m \chi_{[a, b]}} f=m(a) S_{[a, b]} f+\int_{a}^{b}\left(S_{[t, b]} f\right) m^{\prime}(t) d t .
$$

\section{TWO KEY LEMMAS}

Given a dyadic lattice $\mathscr{D}$ in $\mathbb{R}$, a weight $w$ and $k \in \mathbb{Z}$, denote

$$
w_{k, \mathscr{D}}=\sum_{I \in \mathscr{D}:|I|=2^{-k}}\langle w\rangle_{I} \chi_{I}
$$

Lemma 3.1. Let $w \in A_{2}$. Then $w_{k, \mathscr{D}} \in A_{2}$ and

$$
\left[w_{k, \mathscr{D}}\right]_{A_{2}} \leqslant 9[w]_{A_{2}} \text {. }
$$

Also, for two arbitrary dyadic lattices $\mathscr{D}$ and $\mathscr{D}^{\prime}$,

$$
\left[w_{k, \mathscr{D}},\left(\left(w^{-1}\right)_{k, \mathscr{D}^{\prime}}\right)^{-1}\right]_{A_{2}} \leqslant 9[w]_{A_{2}} .
$$

Proof. Denote $u=w_{k, \mathscr{D}}$ and $\mathcal{P}_{k}=\left\{I \in \mathscr{D}:|I|=2^{-k}\right\}$. Take an arbitrary interval $J \subset \mathbb{R}$. Notice that

$$
\langle u\rangle_{J}=\frac{1}{|J|} \sum_{I \in \mathcal{P}_{k}: I \cap J \neq \varnothing} \frac{|I \cap J|}{|I|} \int_{I} w .
$$

Next, by Hölder's inequality,

$$
|I|^{2} \leqslant\left(\int_{I} w\right)\left(\int_{I} w^{-1}\right)
$$

which implies

$$
\begin{aligned}
\left\langle u^{-1}\right\rangle_{J} & =\frac{1}{|J|} \sum_{I \in \mathcal{P}_{k}: I \cap J \neq \varnothing}|I \cap J| \frac{|I|}{\int_{I} w} \\
& \leqslant \frac{1}{|J|} \sum_{I \in \mathcal{P}_{k}: I \cap J \neq \varnothing} \frac{|I \cap J|}{|I|} \int_{I} w^{-1} .
\end{aligned}
$$

Denote

$$
J^{*}=\bigcup_{I \in \mathcal{P}_{k}: I \cap J \neq \varnothing} I
$$

If $|J|>2^{-k}$, then $\left|J^{*}\right| \leqslant 3|J|$, and hence, by (3.3) and (3.4),

$$
\langle u\rangle_{J} \leqslant \frac{1}{|J|} \int_{J^{*}} w \leqslant 3\langle w\rangle_{J^{*}} \text { and }\left\langle u^{-1}\right\rangle_{J} \leqslant 3\left\langle w^{-1}\right\rangle_{J^{*}} .
$$


Assume that $|J| \leqslant 2^{-k}$. Then $\left|J^{*}\right| \leqslant 2^{-k+1}$. Hence in this case,

$$
\langle u\rangle_{J} \leqslant \frac{1}{|I|} \int_{J^{*}} w \leqslant 2\langle w\rangle_{J^{*}} \text { and }\left\langle u^{-1}\right\rangle_{J} \leqslant 2\left\langle w^{-1}\right\rangle_{J^{*}},
$$

which along with (3.5) implies (3.1).

The proof of (3.2) is identically the same. Denote $v=\left(\left(w^{-1}\right)_{k, \mathscr{D}^{\prime}}\right)^{-1}$. If $|J|>2^{-k}$, then by (3.5),

$$
\langle u\rangle_{J} \leqslant 3\langle w\rangle_{J^{*}} \text { and }\left\langle v^{-1}\right\rangle_{J} \leqslant 3\left\langle w^{-1}\right\rangle_{J^{*}}
$$

Similarly, if $|J| \leqslant 2^{-k}$, then

$$
\langle u\rangle_{J} \leqslant 2\langle w\rangle_{J^{*}} \text { and }\left\langle v^{-1}\right\rangle_{J} \leqslant 2\left\langle w^{-1}\right\rangle_{J^{*}}
$$

which along with the previous estimate proves (3.2).

Define the operator $T_{m \chi_{[a, b]}}$ by $\left(T_{m \chi_{[a, b]}} f\right)^{\wedge}=m \chi_{[a, b]} \hat{f}$. In the lemma below we use the same notation $u_{k, \mathscr{D}}$ as in Lemma 3.1.

Lemma 3.2. Assume that $m$ is a bounded and differentiable function on $[a, b]$. Then for all $u, v \in A_{2}$,

$$
\left\|T_{m \chi_{[a, b]}} f\right\|_{L^{2}\left(u_{k, \mathscr{D}}\right)} \leqslant c K(m) N(u, v)\left(2^{-k}(b-a)+1\right)\|f\|_{L^{2}(v)},
$$

where $K(m)=\|m\|_{L^{\infty}}+\int_{a}^{b}\left|m^{\prime}(t)\right| d t$,

$$
N(u, v)=\min \left([u, v]_{A_{2}},\left[u_{k, \mathscr{D}}, v\right]_{A_{2}}\right)^{1 / 2}\left([u]_{A_{2}}^{1 / 2}+[v]_{A_{2}}^{1 / 2}\right)
$$

and $c>0$ is an absolute constant.

Proof. Let $t \in[a, b)$. Take an arbitrary $I \in \mathscr{D}$ with $|I|=2^{-k}$. Notice that

$$
\left\|S_{[t, b]} f\right\|_{L^{\infty}} \leqslant(b-a)\|f\|_{L^{1}} .
$$

Therefore, for all $x, y \in I$,

$$
\begin{aligned}
& \left|S_{[t, b]} f(y)\right| \leqslant(b-a) \int_{3 I}|f|+\left|S_{[t, b]}\left(f \chi_{\mathbb{R} \backslash 3 I}\right)(y)\right| \\
& \leqslant 3(b-a) 2^{-k} M f(x)+\left|S_{[t, b]}\left(f \chi_{\mathbb{R} \backslash 3 I}\right)(y)\right| .
\end{aligned}
$$

Applying (2.5) yields

$$
\begin{aligned}
\left|S_{[t, b]}\left(f \chi_{\mathbb{R} \backslash 3 I}\right)(y)\right| & \leqslant\left|H \mathcal{M}_{-t}\left(f \chi_{\mathbb{R} \backslash 3 I}\right)(y)\right| \\
& +\left|H \mathcal{M}_{-b}\left(f \chi_{\mathbb{R} \backslash 3 I}\right)(y)\right| .
\end{aligned}
$$

For every $t \in[a, b]$,

$$
\begin{aligned}
& \left|H \mathcal{M}_{-t}\left(f \chi_{\mathbb{R} \backslash 3 I}\right)(y)-H \mathcal{M}_{-t}\left(f \chi_{\mathbb{R} \backslash 3 I}\right)(x)\right| \\
& \leqslant c|I| \int_{\mathbb{R} \backslash 3 I}|f(\xi)| \frac{1}{|x-\xi|^{2}} d \xi \leqslant c M f(x) .
\end{aligned}
$$


Further,

$$
\begin{aligned}
\left|H \mathcal{M}_{-t}\left(f \chi_{\mathbb{R} \backslash 3 I}\right)(x)\right| & \leqslant\left|H \mathcal{M}_{-t}\left(f \chi_{\mathbb{R} \backslash[x-|I| / 2, x+|I| / 2]}\right)(x)\right| \\
& +\left|H \mathcal{M}_{-t}\left(f \chi_{3 I \backslash[x-|I| / 2, x+|I| / 2]}\right)(x)\right| \\
& \leqslant H^{\star} \mathcal{M}_{-t} f(x)+c M f(x),
\end{aligned}
$$

which, combined with (3.6), (3.7) and (3.8), implies

$$
\left|S_{[t, b]} f(y)\right| \leqslant H^{\star} \mathcal{M}_{-b} f(x)+H^{\star} \mathcal{M}_{-t} f(x)+\left(3(b-a) 2^{-k}+c\right) M f(x) .
$$

From this and from (2.6), for all $x, y \in I$ we have

$$
\left|T_{m \chi_{[a, b]}} f(y)\right| \leqslant c K(m) \mathcal{T}(f)(x)+\int_{a}^{b} H^{\star} \mathcal{M}_{-t} f(x)\left|m^{\prime}(t)\right| d t,
$$

where

$$
\mathcal{T}(f)(x)=H^{\star} \mathcal{M}_{-b} f(x)+H^{\star} \mathcal{M}_{-a} f(x)+\left(2^{-k}(b-a)+1\right) M f(x) .
$$

Therefore,

$$
\frac{1}{|I|} \int_{I}\left|T_{m \chi_{[a, b]}} f\right|^{2} \leqslant \inf _{I}\left(c K(m) \mathcal{T}(f)+\int_{a}^{b} H^{\star} \mathcal{M}_{-t} f\left|m^{\prime}(t)\right| d t\right)^{2}
$$

Hence, applying Minkowski's inequality and using (2.4), we obtain

$$
\begin{aligned}
& \left\|T_{m \chi_{[a, b]}} f\right\|_{L^{2}\left(u_{k, \mathscr{D}}\right)} \leqslant\left\|c K(m) \mathcal{T}(f)+\int_{a}^{b} H^{\star} \mathcal{M}_{-t} f\left|m^{\prime}(t)\right| d t\right\|_{L^{2}(u)} \\
& \leqslant c K(m)\|\mathcal{T}(f)\|_{L^{2}(u)}+\int_{a}^{b}\left\|H^{\star} \mathcal{M}_{-t} f\right\|_{L^{2}(u)}\left|m^{\prime}(t)\right| d t \\
& \leqslant c K(m)\left(2^{-k}(b-a)+1\right)[u, v]_{A_{2}}^{1 / 2}\left([u]_{A_{2}}^{1 / 2}+[v]_{A_{2}}^{1 / 2}\right)\|f\|_{L^{2}(v)} .
\end{aligned}
$$

On the other hand, (3.9) also implies

$$
\left\|T_{m \chi_{[a, b]}} f\right\|_{L^{2}\left(u_{k, \mathscr{D}}\right)} \leqslant\left\|c K(m) \mathcal{T}(f)+\int_{a}^{b} H^{\star} \mathcal{M}_{-t} f\left|m^{\prime}(t)\right| d t\right\|_{L^{2}\left(u_{k, \mathscr{D}}\right)} .
$$

Therefore, by the previous arguments and Lemma 3.1,

$$
\begin{aligned}
& \left\|T_{m \chi_{[a, b]}} f\right\|_{L^{2}\left(u_{k, \mathscr{D}}\right)} \\
& \leqslant c K(m)\left(2^{-k}(b-a)+1\right)\left[u_{k, \mathscr{D}}, v\right]_{A_{2}}^{1 / 2}\left([u]_{A_{2}}^{1 / 2}+[v]_{A_{2}}^{1 / 2}\right)\|f\|_{L^{2}(v)},
\end{aligned}
$$

which completes the proof.

\section{Proof of Theorems 1.1 And 1.2}

The lower bounds for $\alpha_{p}$ and $\beta_{p}$ are explained in the Introduction. Therefore, we are left with establishing the upper bounds. 
Proof of Theorem 1.1. By duality, the estimate

$$
\|S f\|_{L^{p}(w)} \leqslant C[w]_{A_{p}}^{\frac{1}{2} \frac{1}{p-1}+\max \left(1, \frac{1}{p-1}\right)}\|f\|_{L^{p}(w)}
$$

is equivalent to

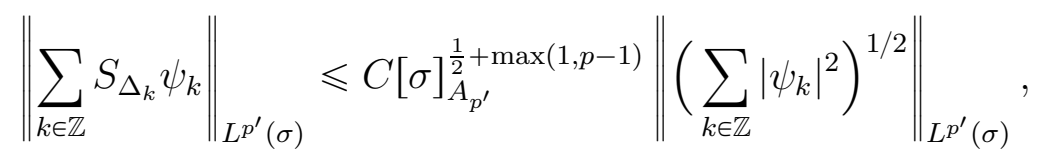

where $\sigma=w^{1-p^{\prime}}$. Changing here $p^{\prime}$ by $p$ and $\sigma$ by $w$, we see that it suffices to prove that

$$
\left\|\sum_{k \in \mathbb{Z}} S_{\Delta_{k}} \psi_{k}\right\|_{L^{p}(w)} \leqslant C[w]_{A_{p}}^{\frac{1}{2}+\max }\left(1, \frac{1}{p-1}\right)\left\|\left(\sum_{k \in \mathbb{Z}}\left|\psi_{k}\right|^{2}\right)^{1 / 2}\right\|_{L^{p}(w)} .
$$

Applying Theorem 2.7 yields

$$
\left\|\sum_{k \in \mathbb{Z}} S_{\Delta_{k}} \psi_{k}\right\|_{L^{p}(w)} \leqslant C[w]_{A_{p}}^{\frac{1}{2}} \sum_{j=1}^{3}\left\|S_{\varphi, \mathscr{D}^{(j)}}\left(\sum_{k \in \mathbb{Z}} S_{\Delta_{k}} \psi_{k}\right)\right\|_{L^{p}(w)} .
$$

Therefore, by Theorem 2.8, (4.2) will follow from

$$
\left\|S_{\varphi, \mathscr{D}}\left(\sum_{k \in \mathbb{Z}} S_{\Delta_{k}} \psi_{k}\right)\right\|_{L^{2}(w)} \leqslant C[w]_{A_{2}}\left\|\left(\sum_{k \in \mathbb{Z}}\left|\psi_{k}\right|^{2}\right)^{1 / 2}\right\|_{L^{2}(w)} .
$$

Using that $\operatorname{supp} \widehat{\varphi_{2^{-k}}} \subset\left\{\xi: 2^{k-1} \leqslant|\xi| \leqslant 2^{k+1}\right\}$, we have

$$
\left(\sum_{j \in \mathbb{Z}} S_{\Delta_{j}} \psi_{j}\right) * \varphi_{2^{-k}}=\left(S_{\Delta_{k-1}} \psi_{k-1}+S_{\Delta_{k}} \psi_{k}\right) * \varphi_{2^{-k}}
$$

which implies

$$
\begin{aligned}
& S_{\varphi, \mathscr{D}}\left(\sum_{j \in \mathbb{Z}} S_{\Delta_{j}} \psi_{j}\right)(x)^{2} \\
& =\sum_{k \in \mathbb{Z}} \sum_{I \in \mathscr{D}: \ell_{I}=2^{-k}}\left(\frac{1}{|I|} \int_{I}\left|\left(S_{\Delta_{k-1}} \psi_{k-1}+S_{\Delta_{k}} \psi_{k}\right) * \varphi_{2^{-k}}\right|^{2}\right) \chi_{I}(x) .
\end{aligned}
$$

Hence, in order to prove (4.3), it suffices to establish that for every $k \in \mathbb{Z}$

$$
\left\|\left(S_{\Delta_{k-1}} f\right) * \varphi_{2^{-k}}\right\|_{L^{2}\left(w_{k, \mathscr{D}}\right)} \leqslant C[w]_{A_{2}}\|f\|_{L^{2}(w)}
$$

and

$$
\left\|\left(S_{\Delta_{k}} f\right) * \varphi_{2^{-k}}\right\|_{L^{2}\left(w_{k, \mathscr{D}}\right)} \leqslant C[w]_{A_{2}}\|f\|_{L^{2}(w)} .
$$

Since

$$
\left(\left(S_{\Delta_{k-1}} f\right) * \varphi_{2^{-k}}\right)^{\Upsilon}(\xi)=\widehat{\varphi}\left(2^{-k} \xi\right) \chi_{\left\{2^{k-1} \leqslant|\xi| \leqslant 2^{k}\right\}} \widehat{f}(\xi),
$$


(4.4) is an immediate corollary of Lemma 3.2 (applied in the case of equal weights). Estimate (4.5) follows in the same way. Notice that the constants $C$ in (4.4) and (4.5) can be taken as

$$
C=c\left(\|\hat{\varphi}\|_{L^{\infty}}+\int_{1 / 2 \leqslant|\xi| \leqslant 2}\left|(\widehat{\varphi})^{\prime}(\xi)\right| d \xi\right)
$$

with some absolute $c>0$.

Remark 4.1. There is a minor inaccuracy in the proof, namely, applying Theorem 2.7, we have used that $\sum_{k \in \mathbb{Z}} S_{\Delta_{k}} \psi_{k} \in L^{p}(w)$ as an a priori assumption. This point can be fixed in several ways. First, by [15], $f \in L^{p}(w)$ implies $S f \in L^{p}(w)$ for $w \in A_{p}$ for all $1<p<\infty$. By duality, this means that $\left(\sum_{k \in \mathbb{Z}}\left|\psi_{k}\right|^{2}\right)^{1 / 2} \in L^{p}(w)$ implies $\sum_{k \in \mathbb{Z}} S_{\Delta_{k}} \psi_{k} \in L^{p}(w)$.

However, one can avoid the use of [15] as follows. Defining

$$
S_{N} f=\left(\sum_{k=-N}^{N}\left|S_{\Delta_{k}} f\right|^{2}\right)^{1 / 2}
$$

we have that (4.1) with $S_{N} f$ instead of $S f$ is equivalent to (4.2) with $\sum_{k=-N}^{N} S_{\Delta_{k}} \psi_{k}$ on the left-hand side. But the fact that $\sum_{k=-N}^{N} S_{\Delta_{k}} \psi_{k} \in$ $L^{p}(w)$ follows immediately from (2.5). The rest of the proof is exactly the same, and we obtain (4.1) with $S_{N} f$ instead of $S f$ with the corresponding constant independent of $N$. Letting $N \rightarrow \infty$ yields the desired bound for $S$.

Remark 4.2. Theorem 1.1 in the case $p=2$ says that

$$
\|S\|_{L^{2}(w) \rightarrow L^{2}(w)} \leqslant C[w]_{A_{2}}^{3 / 2} .
$$

From this, by Theorem 2.8,

$$
\|S\|_{L^{p} \rightarrow L^{p}} \leqslant C\|M\|_{L^{p} \rightarrow L^{p}}^{3 / 2} \quad(1<p \leqslant 2) .
$$

Since $\|M\|_{L^{p} \rightarrow L^{p}} \simeq \frac{1}{p-1}$ for $1<p \leqslant 2$, we obtain the sharp upper bound

$$
\|S\|_{L^{p} \rightarrow L^{p}} \leqslant \frac{C}{(p-1)^{3 / 2}} \quad(1<p \leqslant 2)
$$

found by J. Bourgain [3].

Proof of Theorem 1.2. Using the fact that

$$
\left\|T_{m}\right\|_{L^{p}(w) \rightarrow L^{p}(w)}=\left\|T_{m}\right\|_{L^{p^{\prime}}(\sigma) \rightarrow L^{p^{\prime}}(\sigma)}
$$

and $[\sigma]_{A_{p^{\prime}}}=[w]_{A_{p}}^{\frac{1}{p-1}}$, it suffices to prove that

$$
\left\|T_{m}\right\|_{L^{p}(w) \rightarrow L^{p}(w)} \leqslant C_{p, m}[w]_{A_{p}}^{\frac{1}{2}+\frac{3}{2} \frac{1}{p-1}} \quad(1<p \leqslant 2) .
$$


By Theorems 2.7 and 2.8, (4.6) will follow from

$$
\left\|S_{\varphi, \mathscr{D}}\left(T_{m} f\right)\right\|_{L^{2}(w)} \leqslant C_{m}[w]_{A_{2}}^{3 / 2}\|f\|_{L^{2}(w)} .
$$

Notice that

$$
\left\|S_{\varphi, \mathscr{D}}\left(T_{m} f\right)\right\|_{L^{2}(w)}=\left(\sum_{k \in \mathbb{Z}} \int_{\mathbb{R}}\left|\left(T_{m} f\right) * \varphi_{2^{-k}}\right|^{2} w_{k, \mathscr{D}} d x\right)^{1 / 2} .
$$

Therefore, by duality, (4.7) is equivalent to

$$
\left\|\sum_{k \in \mathbb{Z}}\left(T_{m} \psi_{k}\right) * \varphi_{2^{-k}}\right\|_{L^{2}\left(w^{-1}\right)} \leqslant C_{m}[w]_{A_{2}}^{3 / 2}\left(\sum_{k \in \mathbb{Z}} \int_{\mathbb{R}}\left|\psi_{k}\right|^{2}\left(w_{k, \mathscr{D}}\right)^{-1} d x\right)^{1 / 2} .
$$

Applying Theorem 2.7 again, we see that the question is reduced to the estimate

$$
\begin{aligned}
& \left(\sum_{k \in \mathbb{Z}}\left\|\left(\sum_{j \in \mathbb{Z}}\left(T_{m} \psi_{j}\right) * \varphi_{2^{-j}}\right) * \varphi_{2^{-k}}\right\|_{L^{2}\left(\left(w^{-1}\right)_{k, \mathscr{D}^{\prime}}\right)}^{2}\right)^{1 / 2} \\
& \leqslant C_{m}[w]_{A_{2}}\left(\sum_{k \in \mathbb{Z}}\left\|\psi_{k}\right\|_{L^{2}\left(\left(w_{k, \mathscr{D}}\right)^{-1}\right)}^{2}\right)^{1 / 2}
\end{aligned}
$$

for some dyadic lattices $\mathscr{D}$ and $\mathscr{D}^{\prime}$.

Since

$$
\left(\sum_{j \in \mathbb{Z}}\left(T_{m} \psi_{j}\right) * \varphi_{2^{-j}}\right) * \varphi_{2^{-k}}=\sum_{j=k-1}^{k+1}\left(T_{m} \psi_{j}\right) * \varphi_{2^{-j}} * \varphi_{2^{-k}}
$$

in order to prove (4.8), it suffices to show that for every $k \in \mathbb{Z}$ and every $j=k-1, k, k+1$,

$$
\left\|\left(T_{m} f\right) * \varphi_{2^{-j}} * \varphi_{2^{-k}}\right\|_{L^{2}\left(\left(w^{-1}\right)_{k, \mathscr{D}^{\prime}}\right)} \leqslant C_{m}[w]_{A_{2}}\|f\|_{L^{2}\left(\left(w_{k, \mathscr{D}}\right)^{-1}\right)} .
$$

By Lemma 3.1,

$$
\left.\left.\left[\left(w^{-1}\right)_{k, \mathscr{D}^{\prime}},\left(w_{k, \mathscr{D}}\right)^{-1}\right)\right]_{A_{2}}^{1 / 2}\left(\left[\left(w^{-1}\right)_{k, \mathscr{D}^{\prime}}\right]_{A_{2}}^{1 / 2}+\left[\left(w_{k, \mathscr{D}}\right)^{-1}\right)\right]_{A_{2}}^{1 / 2}\right) \leqslant c[w]_{A_{2}} .
$$

From this and from Lemma 3.2 we obtain (4.9) with

$$
C_{m}=c C_{\varphi}\left(\|m\|_{L^{\infty}}+\sup _{k \in \mathbb{Z}} \int_{\Delta_{k}}\left|m^{\prime}(t)\right| d t\right),
$$

which completes the proof.

Remark 4.3. As in Remark 4.1, it is not difficult to justify the use of Theorem [2.7. We omit the details. 


\section{Concluding Remarks}

5.1. On the sharpness of $\alpha_{p}$ and $\beta_{p}$. The extrapolation principle explained in the Introduction says that if $\xi_{p}$ is the best possible exponent in $\|T\|_{L^{p}(w) \rightarrow L^{p}(w)} \leqslant C[w]_{A_{p}}^{\xi_{p}}$, then $\xi_{p} \geqslant \max \left(\gamma_{2}, \frac{\gamma_{1}}{p-1}\right)$, where $\gamma_{1}$ and $\gamma_{2}$ are the constants appearing in the endpoint asymptotic relations for $\|T\|_{L^{p} \rightarrow L^{p}}$. In fact, for many particular operators we have that $\xi_{p}=\max \left(\gamma_{2}, \frac{\gamma_{1}}{p-1}\right)$.

Therefore, it is plausible that the upper bounds for $\alpha_{p}$ and $\beta_{p}$ from Theorems 1.1 and 1.2 are not sharp for $p>2$ and $1<p<\infty$, respectively, and it is natural to make the following.

Conjecture 5.1. The best possible exponent $\alpha_{p}$ in

$$
\|S\|_{L^{p}(w) \rightarrow L^{p}(w)} \leqslant C_{p}[w]_{A_{p}}^{\alpha_{p}}
$$

is

$$
\alpha_{p}=\max \left(1, \frac{3}{2} \frac{1}{p-1}\right) \quad(1<p<\infty) .
$$

Conjecture 5.2. The best possible exponent $\beta_{p}$ in

$$
\left\|T_{m}\right\|_{L^{p}(w) \rightarrow L^{p}(w)} \leqslant C_{p, m}[w]_{A_{p}}^{\alpha_{p}}
$$

is

$$
\beta_{p}=\frac{3}{2} \max \left(1, \frac{1}{p-1}\right) \quad(1<p<\infty) .
$$

Observe that by Theorem 2.8, in order to establish Conjectures 5.1 and 5.2, it suffices to show that

$$
\|S\|_{L^{5 / 2}(w) \rightarrow L^{5 / 2}(w)} \leqslant C[w]_{A_{5 / 2}} \text { and }\left\|T_{m}\right\|_{L^{2}(w) \rightarrow L^{2}(w)} \leqslant C_{m}[w]_{A_{2}}^{3 / 2},
$$

respectively.

5.2. Sparse bounds for $S$ and $T_{m}$ ? A family of cubes $\mathcal{S}$ is called sparse if there exist $0<\eta<1$ and a family of pairwise disjoint sets $\left\{E_{Q}\right\}_{Q \in \mathcal{S}}$ such that $E_{Q} \subset Q$ and $\left|E_{Q}\right| \geqslant \eta|Q|$ for all $Q \in \mathcal{S}$. By a sparse bound for a given operator $T$ we mean an estimate of the form

$$
|\langle T f, g\rangle| \leqslant C \sum_{Q \in \mathcal{S}}\langle f\rangle_{r, Q}\langle g\rangle_{s, Q}|Q|,
$$

with suitable $1 \leqslant r, s<\infty$, where $\langle f\rangle_{p, Q}=\left\langle|f|^{p}\right\rangle_{Q}^{1 / p}$, and $\mathcal{S}$ is a sparse family.

Sparse bounds have become a powerful tool for obtaining sharp quantitative weighted estimates in recent years (see, e.g., [2, 5, 17]). Therefore it would be natural to try to attack Conjectures 5.1 and 5.2 by means of the corresponding sparse bounds for $S$ and $T_{m}$. 
At this point, we mention that it is not clear to us what is the sparse bound for $S$ leading to Conjecture 5.1. For example, it is plausible that $S$ satisfies

$$
|\langle S f, g\rangle| \leqslant \frac{C}{(r-1)^{1 / 2}} \sum_{Q \in \mathcal{S}}\langle f\rangle_{r, Q}\langle g\rangle_{1, Q}|Q| \quad(1<r \leqslant 2)
$$

but one can show that this estimate leads to the same upper bound for $\alpha_{p}$ as obtained in Theorem 1.1.

Contrary to this, the sparse bound

$$
\left|\left\langle T_{m} f, g\right\rangle\right| \leqslant \frac{C}{(r-1)^{1 / 2}} \sum_{Q \in \mathcal{S}}\langle f\rangle_{r, Q}\langle g\rangle_{r, Q}|Q| \quad(1<r \leqslant 2)
$$

would imply Conjecture 5.2. The technique developed in [21] probably may play an important role in establishing (5.1).

\section{REFERENCES}

[1] O. Bakas, Endpoint mapping properties of the Littlewood-Paley square function, preprint. Available at https://arxiv.org/abs/1612.09573

[2] F. Bernicot, D. Frey and S. Petermichl, Sharp weighted norm estimates beyond Calderón-Zygmund theory, Anal. PDE 9 (2016), no. 5, 1079-1113.

[3] J. Bourgain, On the behavior of the constant in the Littlewood-Paley inequality, Geometric aspects of functional analysis (1987-88), 202-208, Lecture Notes in Math., 1376, Springer, Berlin, 1989.

[4] S.-Y.A. Chang, J.M. Wilson and T. Wolff, Some weighted norm inequalities concerning the Schrödinger operator, Comm. Math. Helv., 60 (1985), 217-246.

[5] J.M. Conde-Alonso, A. Culiuc, F. Di Plinio and Y. Ou, A sparse domination principle for rough singular integrals, Anal. PDE 10 (2017), no. 5, 1255-1284.

[6] D. Cruz-Uribe, J.M. Martell and C. Pérez, Sharp weighted estimates for classical operators, Adv. Math., 229 (2012), no. 1, 408-441.

[7] J. Duoandikoetxea, Fourier analysis. Graduate Studies in Mathematics, 29. American Mathematical Society, Providence, RI, 2001.

[8] J. Duoandikoetxea, Extrapolation of weights revisited: new proofs and sharp bounds, J. Funct. Anal. 260 (2011), no. 6, 1886-1901.

[9] M. Frazier and B. Jawerth, Decomposition of Besov spaces, Indiana Univ. Math. J. 34 (1985), no. 4, 777-799.

[10] M. Frazier, B. Jawerth and G. Weiss, Littlewood-Paley theory and the study of function spaces. CBMS Regional Conference Series in Mathematics, 79, 1991.

[11] T.P. Hytönen, The sharp weighted bound for general Calderón-Zygmund operators, Ann. of Math. 175 (2012), no. 3, 1473-1506.

[12] T.P. Hytönen and M.T. Lacey, The $A_{p}-A_{\infty}$ inequality for general CalderónZygmund operators, Indiana Univ. Math. J. 61 (2012), no. 6, 2041-2092.

[13] T.P. Hytönen and C. Pérez, Sharp weighted bounds involving $A_{\infty}$, Anal. PDE 6 (2013), no. 4, 777-818.

[14] T.P. Hytönen, L. Roncal and O. Tapiola, Quantitative weighted estimates for rough homogeneous singular integrals, Israel J. Math., 218 (2017), no. 1, 133164 . 
[15] D.S. Kurtz, Littlewood-Paley and multiplier theorems on weighted $L^{p}$ spaces, Trans. Amer. Math. Soc. 259 (1980), no. 1, 235-254.

[16] A.K. Lerner, Sharp weighted norm inequalities for Littlewood-Paley operators and singular integrals, Adv. Math., 226 (2011), 3912-3926.

[17] A.K. Lerner, A weak type estimate for rough singular integrals, Rev. Mat. Iberoam., to appear. Available at https://arxiv.org/abs/1705.07397

[18] A.K. Lerner and F. Nazarov, Intuitive dyadic calculus: the basics, Expo Math., to appear. Available at http://arxiv.org/abs/1508.05639

[19] T. Luque, C. Pérez and E. Rela, Optimal exponents in weighted estimates without examples, Math. Res. Lett. 22 (2015), no. 1, 183-201.

[20] S. Petermichl, The sharp bound for the Hilbert transform on weighted Lebesgue spaces in terms of the classical $A_{p}$ characteristic, Amer. J. Math. 129 (2007), no. $5,1355-1375$.

[21] T. Tao and J. Wright, Endpoint multiplier theorems of Marcinkiewicz type, Rev. Mat. Iberoam. 17 (2001), no. 3, 521-558.

[22] M. Wilson, Weighted norm inequalities for the continuous square functions, Trans. Amer. Math. Soc., 314 (1989), 661-692.

[23] M. Wilson, Weighted Littlewood-Paley theory and exponential-square integrability. Lecture Notes in Mathematics, 1924. Springer, Berlin, 2008.

[24] M. Wilson, How fast and in what sense(s) does the Calderón reproducing formula converge?, J. Fourier Anal. Appl. 16 (2010), no. 5, 768-785.

Department of Mathematics, Bar-Ilan University, 5290002 Ramat GAN, ISRAEL

E-mail address: lernera@math.biu.ac.il 